Prefecture, and again in 1965, in the Agano River basin of Niigata Prefecture. Design: The autopsy cases concerned with MD are stored in Kumamoto University (KU) School of Medicine and Brain Research Institute and Niigata University (NU), numbering 450 and 30 case, respectively. These cases were again reexamined at the National Institute for MD.

Results: There are four types of pathological changes in the KU cases: 1) acute adult cases, 2) chronic adult cases, 3) infantile cases, and 4) fetal cases. There are three types of NU cases but no fetal cases. Acute cases showed severe damage to the central nervous system, but the peripheral sensory nerves were not observed in detail. Chronic adult cases showed mild lesions of both the central nervous system and the peripheral sensory nerves. Infantile cases displayed the most severe lesions not only in the central nervous system (spongy state), but also in the peripheral sensory nerves. Hypoplasia was characteristic in the central nervous system of fetal cases, while the peripheral nerves showed only mild lesions. Mercury histochemistry revealed the levels of inorganic mercury in the tissues of autopsy cases to be around $0.2 \mathrm{ppm}$.

Conclusions: Autopsy cases have been reported around the world, from pharmaceutical methylmercury ( $\mathrm{MeHg}$ ) poisoning in England, to $\mathrm{MeHg}$-contaminated bread in Iraq, to pigs poisoned by $\mathrm{MeHg}$ in New Mexico (U.S.A.). Though the autopsy cases outside of Japan showed no lesions of peripheral sensory nerves, the characteristic lesions of central nervous system were still present. This discrepancy may possibly arise due to variance in the duration of $\mathrm{MeHg}$-contaminated fish or shellfish intake and also the dose of $\mathrm{MeHg}$. It should also be noted that the peripheral sensory nerves of MD patients showed regeneration over a long time following the injuries. Sensory disturbance in MD patients is one important characteristic symptom, and both the central and peripheral nervous system show signs of damage. It is thus important to clinically confirm the sensory disturbance in patients with $\mathrm{MeHg}$ poisoning from fish or shellfish through lesions of the central nervous system.

\section{Experimental Pathology}

225 HISTOPATHOLOGICAL INJURY IN THE HEART, INTESTINE AND KIDNEY ASSOCIATED WITH $18 \%, 21 \%$ AND $100 \%$ OXYGEN IN THE RESUSCITATION OF HYPOXIC NEWBORN PIGLETS

Yann Brierley, University of Alberta Hospital, Edmonton, AB, Canada

Background: Controversy exists over the optimal oxygen concentration to administer in the resuscitation of hypoxic newborns. Using a hypoxic newborn animal model, our goal was to elucidate the optimal oxygen concentration to be administered during resuscitation, which best minimizes oxygen-derived free radical injury to individual organs.

Design: Newborn piglets (1-3 days old, 1.7-2.5 kg) were anesthetized and instrumented for continuous monitoring. After $2 \mathrm{~h}$ of normocapnic alveolar hypoxia with $15 \%$ oxygen, piglets were block-randomized to receive $1 \mathrm{~h}$ of reoxygenation with $18 \%, 21 \%$, or $100 \%$ oxygen ( $\mathrm{n}=6$ per group), followed by $2 \mathrm{~h}$ at $21 \%$ oxygen. A sham, non-oxygen deprived, group receiving $21 \%$ oxygen was also included $(n=5)$. Four days after the initial hypoxicreoxygenation event, tissue samples of left ventricle, small intestine and right kidney were obtained for histological examination from all four groups. The histologic tissues were independently assessed by two pathologists, who were blinded to the group assignment. Established grading criteria were utilized to stratify the degree of reoxygenation-induced tissue damage.

Results: Hypoxic piglets showed moderate hypoxemia $\left(\mathrm{PaO}_{2} 27-33 \mathrm{mmHg}\right)$, mild metabolic acidosis ( $\mathrm{pH} 7.20-7.24)$, tachycardia and hypotension (44-50 $\mathrm{mmHg}$ ) compared to the sham piglets $(\mathrm{p}<0.05$, ANOVA). Fourteen of the 18 hypoxic-reoxygenated piglets survived. One piglet died of cardiopulmonary arrest in the $18 \%$ reoxygenated group. Three piglets died ( 1 in the $21 \%$ and 2 in the $100 \%$ reoxygenated group) secondary to severe necrotizing enterocolitis, which occurred over 1 to 3 days following the hypoxiareoxygenation event. Those hypoxic piglets resuscitated with $100 \%$ oxygen showed the most cardiac reoxygenation-induced injury, in the form of marked architectural disruption and overt myocardial necrosis. Myocardial injury in the $100 \%$ reoxygenated group was worse than that observed in the sham $(\mathrm{p}<0.05)$, the $18 \%(\mathrm{p}<0.05)$, and the $21 \%(\mathrm{p}=0.21)$ reoxygenated groups (myocardial necrosis in $75 \%, 0 \%, 0 \%$ and $20 \%$ of $100 \%$, sham, $18 \%$ and $21 \%$ groups, respectively, $\mathrm{p}<0.05$, Chi Square test). No statistically significant difference was found in the degree of tissue injury to the small intestines (including the severe necrotizing enterocolitis associated deaths) and kidneys in the sham or in any of the hypoxic-reoxygenated groups.

Conclusion: Among the organs assessed histologically for evidence of reoxygenation damage following a period of moderate normocapnic hypoxia, the heart was the organ most affected. Myocardial injury was most striking in the $100 \%$ reoxygenated group. Therefore, our results indicate that resuscitation with $100 \%$ oxygen, in the setting of neonatal hypoxia, appears sub-optimal as the first line therapy, given the degree of myocardial injury observed.

\section{ONTARIO TUMOUR BANK INITIATIVE AT HAMILTON HEALTH SCIENCES AND THE JURAVINSKI CANCER CENTRE}

Leela Elavathil, Hamilton Health Sciences, Hamilton, ON, Canada; Nanci Howatt; Hal Hirte; Gurmit Singh, Juravinski Cancer Centre, Hamilton, ON, Canada; Brent Zanke, Cancer Care Ontario, Toronto, ON, Canada; Sugy Kodeeswaran, Ontario Cancer Research Network, Toronto, ON, Canada; Amrita Mishra; Alice Lytwyn, Hamilton Health Sciences, Hamilton, ON Canada

Background: The Ontario Cancer Research Network (OCRN) is a program of the Ontario Institute for Cancer Research (OICR). OICR is a not-for-profit organization mandated to support research in developing and testing new cancer therapies. Funding for OICR comes from the Government of Ontario. The Ontario Tumour Bank (OTB) was initiated in September 2004 as a collaborative initiative between the OCRN and 5 leading Ontario healthcare institutions. The OTB is a repository of neoplastic tumour tissue, peripheral blood, and associated clinical information. The contents of the OTB are accessible by academic and industry researchers for translational research focusing on development of diagnostic tools and novel drug therapies. Hamilton Health Sciences (HHS) joined the OTB consortium in February 2005. We report our experience with the program and the results to date.

Design: Representatives from Anatomical Pathology, Surgery, and Nursing formed the local HHS management committee. OCRN provided funding for capital equipmen purchases and salary support for a Clinical Research Coordinator (CRC) and Pathologists' Assistant (PA). Operating costs are recovered from OCRN funding per banked specimen. Anatomical Pathology supervises the tissue and data collection. The CRC reviews the preoperative list of patients from participating surgeons, and obtains consent and a blood sample in the pre-operative clinic. The CRC reviews the patient chart and completes the OTB clinical data collection forms. On the day of surgery, the PA informs the operating room (OR) of consenting patients, and the OR pages the PA when the tissue is resected. The PA has been trained by the pathologist to collect representative fresh tumour tissue, and to request pathologist assistance when needed. Tissue is placed into $2 \mathrm{ml}$ cryovials and frozen in liquid nitrogen within 30 minutes of devitalization. When available, normal adjacent tissue is also sampled. Two formalin fixed paraffin embedded blocks are made for each case. Tissue is not collected if it may compromise pathology assessment. Representative tissue is tested at a central laboratory for DNA and RNA integrity.

Results: Since the inception of OTB, the HHS site has contributed tissue samples, blood and clinical information from $406(26.2 \%)$ of the 1551 consented donors. The top three tumour sites in the OTB are gynecological, lung and breast. Seventy seven percent $(312 / 406)$ of tumours from HHS are gynecological cancers, breast cancers and central nervous system tumors. The mean size of tumour sample in OTB is $250 \mathrm{mg}$. Quality assurance testing has shown that RNA quality from tissue samples, and the DNA quality from buffy coat blood samples, is very good or acceptable in $83 \%$ and $85 \%$, respectively. The website for the OTB was launched in March 2006 (www. ontariotumourbank. ca) with an ongoing call for proposals.

Conclusion: OTB has successfully completed 18 months of tissue and data collection. HHS has substantially contributed to the provincial totals. Surgeons, pathologists and basic scientists at HHS have come together in support of this valuable resource. Opportunities for collaboration and pathology involvement in translation research have increased.

\section{RAPAMYCIN INHIBITS THE SPONTANEOUS FORMATION OF DE} NOVO CANCER IN P53 KNOCKOUT MICE

Andreas Gaumann; Gudrun Koehl; Anna Hoehn; Manuela Kovacs; Hans Schlitt; Edward Geissler, University of Regensburg, Regensburg, Germany

Background: Cancer is a major problem in transplant recipients. Recent data suggest that immunosuppressive drugs like rapamycin (RAPA), or possibly mycophenolate mofetil (MMF), may be capable of reducing the growth of existing tumors. However, little experimental data exists regarding cancer prevention. Here we tested the effects of longterm RAPA, MMF or cyclosporine (CsA) use on spontaneous tumor formation in p53 knock-out $(\mathrm{KO})$ mice.

Design: p53 KO mice received either no treatment, or were treated with RAPA, MMF or CsA at therapeutic levels starting on week 10 after birth. Drugs were fed via custommade mouse-chow formulations to attain typical immunosuppressive doses. Mice were monitored daily and were sacrificed when clinical signs of disease occurred. The experimental endpoint was at week 28 . Tissues from major organs and masses were taken for histologic analysis. Immunohistochemistry confirmed the diagnosis of lymphomas and sarcomas.

Results: All (9/9) untreated mice developed clinically evident tumors by week 28 (mean 19.7 \pm 5.3 ), as confirmed by histology (6 lymphomas, 3 sarcomas). All CsA-treated mice (9/9) also developed clinical tumors by week 28 (mean: $20.2 \pm 5.3 ; 8$ lymphomas, 1 sarcoma). With MMF treatment, $7 / 10$ mice showed clinical evidence of tumor by week 28 (mean: 18.4 $\pm 3.7 ; 3$ lymphomas, 4 sarcomas), however, histology of the lymphatic organs revealed that the remaining 3 mice had subclinical cancer ( 3 lymphomas). In contrast, RAPA treatment resulted in only 3 clinically evident tumors before the experimental endpoint (week 12, 18, 26; all lymphomas), with histology revealing subclinical lymphomas in 3 additional mice, but no evidence of tumor in any of the major organs in 4 animals. Logrank analysis shows a significant decrease in cancer occurrence in the RAPA group $(\mathrm{P}=0.02$ vs. controls); tumor occurrence was not significantly altered in either the CsA or MMF groups.

Conclusions: Our results show that de novo development of cancer is reduced in p53 KO mice under RAPA immunosuppression. Although MMF may reduce de novo tumors appearance slightly, the effect is not significant, and CsA does not affect de novo tumor development in this model. These results are the first to show spontaneous cancers in mice arising from $\mathrm{p} 53$-mutations can be reduced by RAPA immunosuppression.

\section{HLB BLOOD TEST TO EVALUATE THE OXIDATIVE STRESS IN CRONIC} DISEASE

Dary Oliveira, Federal University of Ceara, Fortaleza, Brazil

Background: Pathology caused by, associated with or adjunctive to, reactive oxygen species (ROS) plays a role in virtually all disease states and metabolic dysfunctions Biochemical interactions of these species cause particular "biological markers" which leave characteristic patterns in blood, observed by microscopy.

Design: The aim of this study is to identify the correlation of HLB Blood test (for HeitanLaGarde-Bradford) and other biochemical parameters. Methods include a review of 1000 patients who had a HLB Blood test performed according Bradford Research Institute, admitted to Pathology Institute of Ceara, between 1998 and 2003. A drop of capillary blood was expressed from the tip of the finger and transferred to a glass slide in a series of drops to produce a desired diminishing thickness. The typical morphological stages are shown in the microphotographs (eclipse E-400, Y-Fl-Epi-Fluorescence attachment).

Results: Results revealed good correlation between the HLB test in 82 per cent of cancer 
cases, 86 per cent of stress cases, 88 per cent of hormonal disturbances and 90 per cent of degenerative diseases.

Conclusion: In conclusion, the HLB test appears to be a good method to evaluate the effects of free radicals in many pathological situations.

\section{RENOPROTECTIVE EFFECTS OF ACE INHIBITION IN ALLOXAN-} INDUCED HYPERCHOLESTEROLEMIC RABBITS

Daniel Pomaro, Federal University of São Paulo, São Paulo, Brazil; Marcello Franco, Federal University of São Paulo - Paulista Medical School - Department of Pathology, São Paulo, Brazil; Thiago Simão Gomes; Maria Cristina O. Izar; Francisco Fonseca; Silvia Ihara, Federal University of São Paulo, São Paulo, Brazil

Background: Recently, our group performed a study investigating the effects of the angiotensin-converting enzyme inhibitor (ACEI) quinapril on the development of atherosclerosis in rabbits with hypercholesterolemia and alloxan-induced diabetes ( $\mathrm{J}$ Cardiovasc Pharmacol 2005; 45:295-300), and we verified that higher glucose levels may abolish the protective anti-atherosclerotic effect of ACE inhibitors on atherosclerosis development.

Design: Diabetes was induced in 49 New Zealand male rabbits with a single dose of alloxan $(100 \mathrm{mg} / \mathrm{Kg}$, i.v.) and, according to plasma glucose levels after one week, the animals were divided in two groups $(>250 \mathrm{mg} / \mathrm{dL}$ GI, GII and $<250 \mathrm{mg} / \mathrm{dL}$ GII and GIV). All animals received a $0.5 \%$ cholesterol-enriched diet for 12 weeks. Each group was randomly assigned to receive (GII and GIV) or not (GI and GIII) quinapril (30 mg/day) added to the hypercholesterolemic diet. Histomorphometry of the aortas (Intima/media ratio (I/M) and the percent of intima area occupied by macrophages (\%)) and kidneys (glomerular area, GA $(\mu \mathrm{m} 2)$ and the percent of glomerular area occupied by macrophages $(\%)$ ) were analyzed. ACE activity was measured by spectrofluorimetric assay.

Results: Reduction of I/M ratio of aortas was observed only in the quinapril-treated animals that presented low glucose levels (GIV $0.12 \pm 0.03 *$ vs. GI $0.34 \pm 0.01$, GII $0.27 \pm 0.05$ and GIII $0.51 \pm 0.10 ; \mathrm{p}<0.01$ ), in spite of similar ACE activity in all treated groups (GI 4.06 \pm 0.38 , GII $2.43 \pm 0.23 *$, GIII $3.69 \pm 0.27$ and GIV $2.35 \pm 0.32 * ; p<0.05$ ). Macrophage distribution in intima layer was not different between groups. In the kidneys, the GA (GI 7190 \pm 348 , GII $7546 \pm 601$, GIII $6656 \pm 577$, GIV $7575 \pm 440$ ) was not different among groups. However, quinapril-treated animals had a significant reduction in the area occupied by macrophages (GI $3.14 \pm 1.14$, GII $0.72 \pm 0.08 \dagger$, GIII $2.44 \pm 0.54$, GIV $0.78 \pm 0.11 \dagger, \mathrm{p}<0.05$ ), regardless of glucose levels.

Conclusion: In alloxan-induced diabetes ACE inhibition may exert renoprotective effects by reduction of glomerular macrophage accumulation. Conversely, in aortas this atheroprotective effect may be dependent on glucose levels.

\section{ESTIMATION OF NUCLEAR DENSITY INDEPENDENT OF THICKNESS OF SPECIMEN}

Masanobu Takahashi; Takashi Yoshimi, Shibaura Institute of Technology, Saitama-city, Saitama, Japan; Masayuki Nakano, Chiba Medical Center, Chiba-city, Chiba, Japan

Background: The nuclear density (the number of nuclei per unit area), which is related to cellularity, is one of important features in histopathologic diagnosis. Nuclear density, however, depends on both the thickness of a specimen and the diameter of nuclei. This dependency originates from using two dimensional (2-D) nuclear density instead of 3-D density because nuclear density itself should originally be three dimensional (number of nuclei per unit volume). So, we developed a method to estimate 3-D nuclear density from an observed 2-D microscopic image.

Design and Results: In the estimation, nuclei were assumed to be mostly spherical, and the small nuclei having cross sections less than $25 \%$ of the average nuclei were ignored. Assuming the field of view is large enough and the average diameter $\mathrm{D}_{\mathrm{av}}$ of nuclei is known, the 3-D nuclear density can be calculated using the following equation, where $\mathrm{T}$ is the thickness of a specimen. 3-D nuclear density $=2-\mathrm{D}$ nuclear density $/\left(\mathrm{T}+0.87 \mathrm{D}_{\mathrm{av}}\right)(1)$ In the actual diagnosis, however, the field of view is limited depending on the magnification factor of an objective lens. The average diameter $D_{a v}$ of nuclei is also unknown. In such a case, the 3-D nuclear density can be estimated probabilistically using Bayes estimation. The 3-D nuclear density and other unknown parameters, the average diameter $\mathrm{D}_{\mathrm{av}}$ and the variance of diameters, are estimated using features extracted from a microscopic image. This time, histogram of nuclear areas was used as the features. Computer simulation was carried out using a hypothetical hepatic histopathologic specimen in which nuclei with $D_{a v}$ of 7.0im distribute randomly with a pre-determined 3-D nuclear density. Simulation results showed that the estimated 3-D nuclear density (the expectation value) was almost the same as the pre-determined 3-D density. The accuracy $(90 \%$ confidence interval) was $+-16 \%$ when a $x 40$ objective lens (field of view: $0.071 \mathrm{~mm}^{2}$ ) was used. The accuracy was improved up to $+-9 \%$ and $+-6 \%$ when a $\times 20$ and a $\times 10$ objective lenses were used, respectively. The accuracy is improved by using an objective lens with a smaller magnification factor because the field of view becomes larger. The average diameter $D_{\text {vy }}$ was also estimated to be $7.0 \mathrm{im}+-0.2 \mathrm{im}$ (x40 objective lens). Experiments were also carried out using microscopic images of a real hepatic histopathologic specimen (hematoxylin-eosin stained, 3-ìm thick). The accuracy $(90 \%$ confidence interval) of the estimated 3-D density was $+-15 \%$ and + $7 \%$ when a $x 40$ and a $\times 20$ objective lenses were used, respectively. The results well agreed with the simulation.

Conclusion: Developed method enables the estimation of 3-D nuclear density from a 2-D microscopic image usually used in the diagnosis. The average and the variance of nuclear diameters can also be estimated. The 3-D nuclear density will be useful as an absolute feature which is independent of both the thickness of a specimen and the diameter of nuclei.

Acknowledgement: This research was partially supported by KAKENHI (16500302).

\section{Forensic}

\section{PATHOMORPHOLOGY OF IMMUNE-ENDOCRINE SYSTEM DURING} CHRONIC DRUG ADDICTION

Adalat Hasanov; Vugar Huseynov, Forensic Medical Expertise and Pathologic Anatomy Unification, Baku, Azerbaijan

Background: The morphological features and potential of differential diagnoses of chronic drug addiction have been studied in its different development stages on a basis of analysis of pathomorphological changes occurred in the immune and endocrine system organs.

Design: The bodies of 185 individuals aged between 17 and 40 (164 men and 21 woman), who were subjected to chronic drug addiction, where dissected 104 bodies where pathoanatomical - (33 - septic endocarditis, 18 septicopiemia, 53 tuberculosis, pneumonia etc.). And 81- forensic expertise (57-acute drug poisoning, 24injuires with guns and stabs auto trauma suicide etc. All observations were divided into two groups depending on the period of drug abuse. Chronic drug addicts abusing drugs for less than two years and those abusing drugs from two to ten years. Micro preparations where painted with picrofucsin and sudan-3, with the method of hepatocsilin and eosin, orsein, van-Giron, the pearls reaction was carried out. Immune-morphological examinations were also held by using the panel of standard monoclonal antibodies.

Results: During the examinations included in the first group, the morphological changes in the immune and endocrine organs were mainly of reactive-hyper plastic and dystrophic nature and accompanied by acute microcirculatory disturbances. The rapid emigration of T-lymphocytes to bone ILIK in immune system organs, especially in thymus, weakening of mitotic activeness of lymphocytes and strengthening of apoptosis, increase in activeness of B-lymphocytes in other lymphoid tissues were observed. During the examinations in the second group, necrotic, atrophic and sclerotic changes along with the abovementioned pathological changes were discovered in the immune and endocrine organs. This means development of secondary B- and T-cell type immune deficiency situation in patients.

Conclusion: The changes happened in the neuro-endocrine-immune system during chronic drug addiction develop within realization of a common adaptation syndrome like during all other strong stresses. The intravenous drug addiction, which has high toxic features, this time, plays the role of a stressing factor. The great role of handmade drugs in the development of chronic stress reaction in organism as well as the additional high toxic substances in the composition of solution injected intravenous in the organism are mentioned. It is necessary to consider the secondary immune deficiency situations developing during chronic drug addiction as the complex problem of functional disturbances of neuro-endocrine and immune system.

232 HISTOPATHOLOGICAL STUDY CONTRIBUTION FOR DIAGNOSIS OF FIREARMS HUMAN SKIN WOUNDS HISTOPATHOLOGICAL STUDY FOR DIAGNOSIS OF HUMAN SKIN FIREARM WOUNDS

Solange Pires D Avila; Marcus Baptista, Faculdade de Medicina de São José do Rio Preto, São José Rio Preto, Brazil

Background: Fatal human firearm injuries are the third leading cause of deaths in Brazil This epidemiological data observation in death certificates is only surpassed by cardiac and cerebral vascular disease. Justice and Forensic Medicine have interest in these human injury study. For the objective of Justice the cause of death as well the description of the wounds in the human being are provided to the Forensic Physician. In cases of death associated with firearms, the medical examiner has to determine if the orifice from the projectile in the body is of entrance or exit. This determination is very important according to the juducial and proceeding point of view. The firearm projectiles cause in the human skin several typical wounds; however, the shot direction or if the wound occurred due to the entrance or the exit of the projectile are not always determined macroscopically by the medical examiner. Therefore, when necessary, other means should be taken to provide an accurate diagnosis.

Design: To examine in the human skin, histopathological characteristics to determine entrance or exit firearm wounds.

Results: Macro and microscopically, 14 entrance and their 14 exit fatal human skin firearm wound specimens were analyzed. Coagulation necrosis of keratinocytes associated with subepidermal fissure (effects of burn injuries) was a characteristic feature observed only for the entrance orifice. Presence of adipose, muscle and bone tissues in the dermis was a characteristic feature observed only for the exit orifice. Literature of comparable data were reviewed and are similar with this observation.

Conclusion: The histopathological human skin analysis provides important information for the Medical Examiner, and will help the diagnosis of the entrance and the exit firearm wounds for Justice.

233 SUDDEN DEATH PREVALENCE ANALYSIS AND ASSOCIATED RISK FACTORS: STUDY WITH 2056 PATIENTS SUBMITTED TO NECROPSY

Luciana Martins Reis; Jose Antonio Cordeiro; Patricia Maluf Cury, Faculdade de Medicina de Sao Jose do Rio Preto, SJ Rio Preto, Brazil

Background: Sudden death is defined as instantaneous death or until 24 hours after beginning of the signals and symptoms. Objectives: to evaluate the prevalence of sudden death and to correlate with associated risk factors, in autopsies carried through in the Department of Pathology.

Design: The final autopsy reports performed between 1993 and 2002 were analysed and clinical and pathological data were collected.

Results: Of the 2056 autopsies of the period, $753(36,62 \%)$ were of sudden death. The mean age was of 62,16 years, $62.95 \%$ were male, with mean age of 59,9 years and the female mean age was 66,1 years. The main cause of sudden death was acute myocardial infarction $(51,07 \%)$. The main basic disease associated was arteriosclerosis $(40,26 \%)$ and systemic arterial hypertension $(10 \%)$. When correlating the age with the cause of sudden death we observed that the mean age of the patients with intracranial hemorrhage was 